\title{
Experimental Demonstration of Time-Reversal MISO and MIMO Arrays with IEEE 802.11g Devices through a Ventilation Duct Channel
}

\author{
Benjamin E. Henty \\ i5wireless, LLC. \\ Lanham, MD, 20706 \\ Email: henty+vtc@eirp.org
}

\author{
Daniel D. Stancil \\ Carnegie Mellon University \\ Pittsburgh, PA, 15213 \\ Email: stancil@cmu.edu
}

\begin{abstract}
A practical demonstration of IEEE 802.11g transmissions using time-reversal antenna arrays in MISO and MIMO configurations is presented in this work. Throughput measurements indicate significant channel improvement through the use of time-reversal array coefficients and illustrate the viability of increased Access Point (AP) capacity and co-channel interference reduction in multi-user scenarios.
\end{abstract}

\section{INTRODUCTION}

There has been a great deal of recent interest in the application of a technique known as time-reversal for use at electromagnetic, microwave frequencies. Several authors have presented demonstrations of communications at radio frequencies $(\mathrm{RF})$ indicating that the technique is a valid one for use with electromagnetic waves [1-3]. Further, it has been noted that time-reversal can provide a reduction in the delay spread of a communications channel [4]. Additional work with time-reversal has shown it to be a technique with great potential for use in MISO (multiple-input single output) arrays [5,6].

In this work we present a practical demonstration of wireless local area network communications (WLAN) in the form of IEEE $802.11 \mathrm{~g}$ signals, using a timereversal focusing antenna array. We present throughput performance measurements of actual IEEE $802.11 \mathrm{~g}$ transmissions using MISO and MIMO antenna arrays. For these experiments we have used a closed ventilation duct as a communications channel. There are several advantages to such a channel. First, since the channel is static, it is possible to characterize the channel, perform calculations as necessary for time-reversal and apply the time-reversal to data transmissions, all without concern that the channel may have changed. Further, the highly reverberant nature of the duct channel should allow us to observe any improvement in performance due to a reduction in the delay spread of the channel from time-reversal coefficients. Lastly, significant work (see for examples $[7,8]$ ) has been undertaken to realize ventilation ducts as a means to distribute wireless signals in a building. As a result, this work aids in exploring the use of time-reversal arrays for use in the wireless communications through ventilation duct application.

\section{Time-Reversal Measurements}

\section{A. Experimental Setup}

Our measurement setup is shown Fig. 1. Five cylindrical spiral duct segments, $0.3048 \mathrm{~m}$ in diameter and totaling $15.2 \mathrm{~m}$ in length were used for the measurements and had metal endcaps in place. Quarter wavelength monopole antennas were inserted into the ventilation duct for all transmissions. A four element, axial antenna array with element spacings of one wavelength (12.24 $\mathrm{cm}$ ) was installed halfway between two single element antennas. In order to apply time-reversal coefficients, we used vector modulators with the antenna array. We used Herley Farmingdale 7122 vector modulators which use digital input signals and are interfaced to a computer parallel port via a custom circuit board and controlled via custom MATLAB ${ }^{\circledR}$ software. Each modulator is bidirectional and multiplies the RF signals passing through the modulator in either direction by a complex exponential, $A e^{j \theta}$. The modulators are calibrated to provide amplitude coefficients, $A$, ranging from 0 to $-70 \mathrm{~dB}$ and phase shifts, $\theta$, of 0 to 360 degrees with an accuracy of $\pm 0.2 \mathrm{~dB}$ and \pm 5 degrees over the magnitude and phase shifts and frequency ranges used in the experiment.

We wish to attempt two simultaneous connections via time-reversal, so we have placed two Linksys WRT54G, IEEE 802.11g Access Points (APs) inside metal project boxes. We filled the empty space inside these boxes with RF absorbing material and connected one antenna port to an SMA passthrough connector on the boxes. Power and ethernet cables were also passed through a hole in the boxes. The holes and all seams were sealed with aluminum and copper tape. When the SMA passthrough was terminated with a matched, $50 \Omega$ load, the APs could not be detected with a wireless LAN card, even right next to the boxes. Two laptops, 1 and 2, were connected via ethernet cables, one to each AP. These laptops were used for throughput measurements. Two other laptops, 
labeled 3 and 4, with Buffalo Wireless Air Station, WLICB-G54A, IEEE 802.11g NICs (Network Interface Cards) were used to connect to the duct endpoints and were also used for throughput measurements. A final two laptops, labeled 5 and 6 in Fig. 1, were used to control the settings of the vector modulators, which are labeled "IQ" in Fig. 1 because they can also be conceptualized as IQ modulators. Lastly, $60 \mathrm{~dB}$ attenuators were added to the outputs of the APs. These attenuators were added to shift the total pathloss down to a level that allowed the IEEE 802.11 cards to use a moderate to low transmit rate. In this way any improvements due to time-reversal would be apparent. The attenuators also serve to reduce the power to a level that will not damage the vector modulators and also prevents the APs from detecting one another via leakage in the power divider modules. We also note that each of the cables used in parallel paths were of the same type (RG-142) and of matched length. Paired power dividers were also of the same type. These techniques were used to ensure each path through the RF circuitry had as close as possible the same phase and magnitude response.

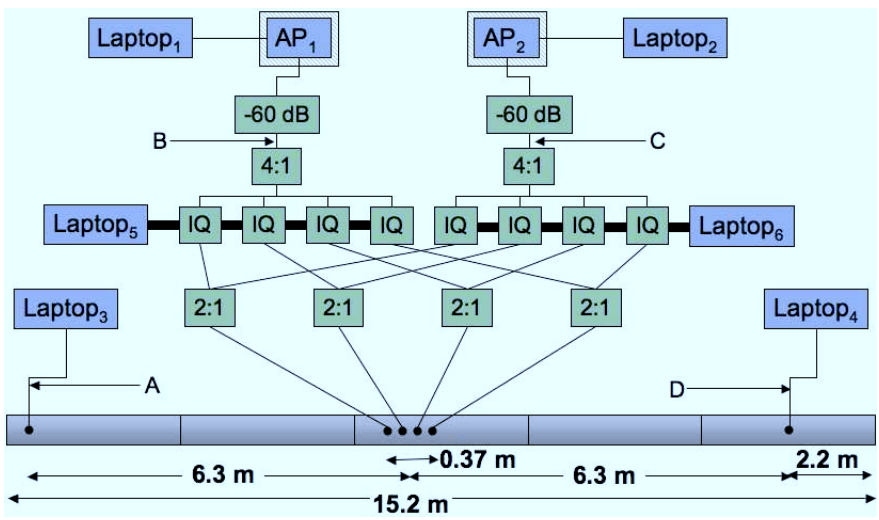

Fig. 1. Measurement setup of time-reversal arrays for IEEE 802.11 measurements.

In order to apply time-reversal coefficients, we needed to first characterize the full $H$ matrix. To measure each component of the 4 by 2 matrix, we used a vector network analyzer (VNA). Rather than measure just the $H$ matrix of the duct, we characterized each component including the vector modulators. This was done by connecting the network analyzer to the insertion points $A$, $B, C$ and $D$ indicated in Fig. 1 . Since our VNA can only characterize a two port network, we connect the VNA pairwise to the ports $A-B, A-C, D-B$ and $D-C$. For each connection, we use the vector modulators to characterize the 4 by $2 \mathrm{H}$ matrix. This is accomplished by setting all of the vector modulators to a maximum attenuation and then setting a single modulator in one of the RF paths to a known, zero magnitude, zero degree reference point. In this manner each of the paths through the vector modulators and the full 4 by $2 H$ matrix through the ventilation duct could be characterized. As part of this process the magnitude and phase responses at the center frequencies of the first six IEEE 802.11g channels were recorded to use for computing time-reversal coefficients.

\section{B. MISO Time-Reversal Focusing}

After characterizing the ventilation duct $H$ matrix with a VNA, we then connected the laptops shown in Fig. 1. We first attempted time-reversal focusing using a single AP and single user location. This effectively reduces the 4 by $2 H$ matrix to a 4 by 1 matrix. To measure the impact of time-reversal focusing on IEEE 802.11g communications, we have performed throughput measurements over the wireless LAN link using two different antenna array coefficients. The first set of coefficients was simply each vector modulator set to its 0 magnitude, 0 phase position. The second set of coefficients was the appropriate time-reversal coefficient setting on each vector modulator.

The vector modulators are limited in the time-reversal coefficients that they can set. The modulators are attenuate only devices with a range of attenuations and phases that can be applied. One limitation is that to guarantee that any phase can be set, a minimum attenuation of $0.5 \mathrm{~dB}$ is required. For this reason, when applying timereversal coefficients, the computed coefficient with the lowest required attenuation was set to $0.5 \mathrm{~dB}$. All other coefficients were scaled appropriately to maintain the correct time-reversal coefficient. This process ensures that the range of attenuations set by the vector modulators (18 $\mathrm{dB}$ in the measurements presented below) and the needed phase shifts are both well within the calibrated ranges of the devices. However, this procedure also prevents the time-reversal signal from being sent with the same transmit power as the equal phase case. While this prevents the comparison from being strictly fair, it is not necessarily unreasonable. While we do not anticipate an actual time-reversal system using the same technique as we use for these measurements, we do expect that the waveform shaping needed for time-reversal will reduce the maximum power output of a practical device somewhat, as is the case in our measurements. Therefore, we allow time-reversal to be at a disadvantage in terms of power, knowing that some limitations will be imposed in a practical deployment.

Throughput measurements in the two cases were performed using the Iperf measurement tool [9], which is an open source, multi-platform software utility. Throughput was measured in both the uplink and downlink directions by running a server and client on each computer as needed. TCP throughput was measured for 60 seconds for each test using a TCP window size of 1 megabyte. Laptops 1 and 3, shown in Fig. 1, were used for the throughput measurements. Measurements were performed at each of the first six IEEE $802.11 \mathrm{~g}$ channel center frequencies: 2.412, 2.417, 2.422, 2.427, 
TABLE I

Throughrut MEASUREMENTS AND CHANNEL StATISTICS FOR 4 BY 1 ANTENNA ARray With EQUal Amplitude AND Time-REversal COEFFICIENTS

\begin{tabular}{|c|c|c|c|c|}
\hline Frequency & $\begin{array}{c}\text { Receive } \\
\text { Power }\end{array}$ & $\begin{array}{c}\text { Gain } \\
\text { Standard } \\
\text { Deviation }\end{array}$ & $\begin{array}{c}\text { Uplink } \\
\text { Through- } \\
\text { put }\end{array}$ & $\begin{array}{c}\text { Downlink } \\
\text { Through- } \\
\text { put }\end{array}$ \\
\hline \hline \multicolumn{5}{|c|}{ Equal Amplitude Coefficients } \\
\hline \hline $2.412 \mathrm{GHz}$ & $-74.6 \mathrm{dBm}$ & $5.6 \mathrm{~dB}$ & $2.9 \mathrm{Mbps}$ & $2.9 \mathrm{Mbps}$ \\
$2.417 \mathrm{GHz}$ & $-75.1 \mathrm{dBm}$ & $5.1 \mathrm{~dB}$ & $2.3 \mathrm{Mbps}$ & $2.1 \mathrm{Mbps}$ \\
$2.422 \mathrm{GHz}$ & $-74.4 \mathrm{dBm}$ & $5.2 \mathrm{~dB}$ & $2.5 \mathrm{MBps}$ & $2.3 \mathrm{Mbps}$ \\
$2.427 \mathrm{GHz}$ & $-73.3 \mathrm{dBm}$ & $5.9 \mathrm{~dB}$ & $3.6 \mathrm{Mbps}$ & $3.3 \mathrm{Mbps}$ \\
$2.432 \mathrm{GHz}$ & $-73.5 \mathrm{dBm}$ & $6.3 \mathrm{~dB}$ & $3.4 \mathrm{Mbps}$ & $3.1 \mathrm{Mbps}$ \\
$2.437 \mathrm{GHz}$ & $-74.0 \mathrm{dBm}$ & $6.3 \mathrm{~dB}$ & $2.2 \mathrm{Mbps}$ & $2.3 \mathrm{Mbps}$ \\
\hline \hline \multicolumn{5}{|c}{ Time-Reversal Coefficients } \\
\hline \hline $2.412 \mathrm{GHz}$ & $-71.9 \mathrm{dBm}$ & $2.5 \mathrm{~dB}$ & $3.7 \mathrm{Mbps}$ & $3.4 \mathrm{Mbps}$ \\
$2.417 \mathrm{GHz}$ & $-71.7 \mathrm{dBm}$ & $2.3 \mathrm{~dB}$ & $4.2 \mathrm{Mbps}$ & $3.9 \mathrm{Mbps}$ \\
$2.422 \mathrm{GHz}$ & $-72.2 \mathrm{dBm}$ & $2.2 \mathrm{~dB}$ & $3.9 \mathrm{Mbps}$ & $3.7 \mathrm{Mbps}$ \\
$2.427 \mathrm{GHz}$ & $-72.5 \mathrm{dBm}$ & $2.0 \mathrm{~dB}$ & $5.9 \mathrm{Mbps}$ & $4.3 \mathrm{Mbps}$ \\
$2.432 \mathrm{GHz}$ & $-72.2 \mathrm{dBm}$ & $1.7 \mathrm{~dB}$ & $4.7 \mathrm{Mbps}$ & $4.5 \mathrm{Mbps}$ \\
$2.437 \mathrm{GHz}$ & $-72.6 \mathrm{dBm}$ & $3.4 \mathrm{~dB}$ & $4.4 \mathrm{Mbps}$ & $4.1 \mathrm{Mbps}$ \\
\hline
\end{tabular}

2.432 and $2.437 \mathrm{GHz}$. The resulting uplink and downlink throughput measurements are shown in Table I. The table also has the calculated receive power and standard deviation of the frequency response for the total 4 by 1 channel. The received power shown is measured by first calculating the sum response of the four duct channels each multiplied by the appropriate equal amplitude or time-reversal coefficients. Then, taking into account the $18 \mathrm{dBm}$ transmit power of the APs and the attenuators added, the expected receive power at the user laptop is computed by integrating over the $16 \mathrm{MHz}$ span around the center frequency. The gain standard deviation shown in the table is computed over the $22 \mathrm{MHz}$ range around the center frequency.

The data in Table I indicates that the time-reversal settings are functioning as we would expect. The standard deviation in the channel response decreased from about $5-6 \mathrm{~dB}$ to $2-3 \mathrm{~dB}$. While the improvement in the channel gain is not the anticipated $6 \mathrm{~dB}$, it does increase somewhat. This is reasonable given the limitations imposed by the vector modulators used. It is gratifying to see that the frequency response improvements do translate into improved throughput. Fig. 2 is a plot of the receive power and throughput values from Table I. The figure suggests that the improvements in throughput observed could be explained solely by the improvement in signal strength due to the time-reversal focusing.

It is somewhat surprising that the single frequency time-reversal coefficient is effective over the range of frequencies that are used by the IEEE $802.11 \mathrm{~g}$ communications. Fig. 3 shows a comparison of the computed frequency responses for the two sets of antenna array coefficients applied for the first center frequency, 2.412 $\mathrm{GHz}$. The figure also shows the computed ideal response if ideal time-reversal coefficients were applied to the antenna array at each frequency, using the same

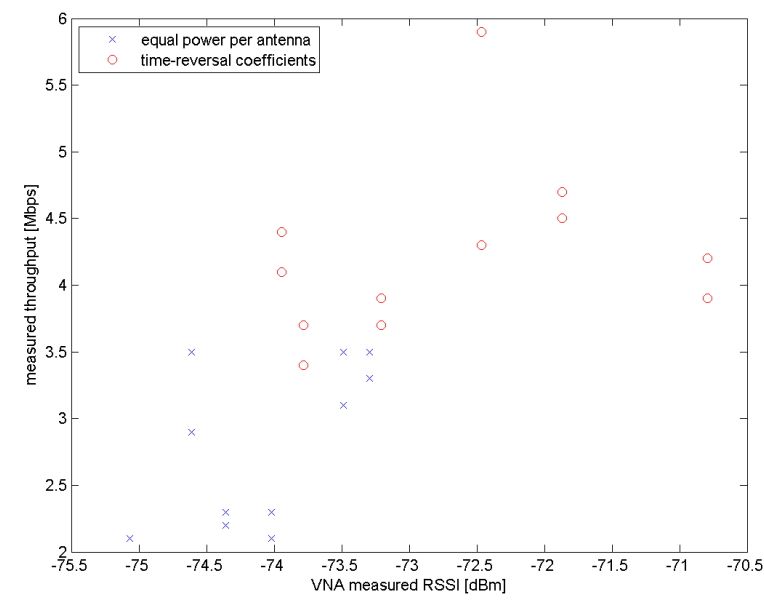

Fig. 2. Uplink and Downlink Throughput versus Signal Strength as Computed from VNA Measured Frequency Responses.

attenuate-only power limitations imposed by the vector modulators.

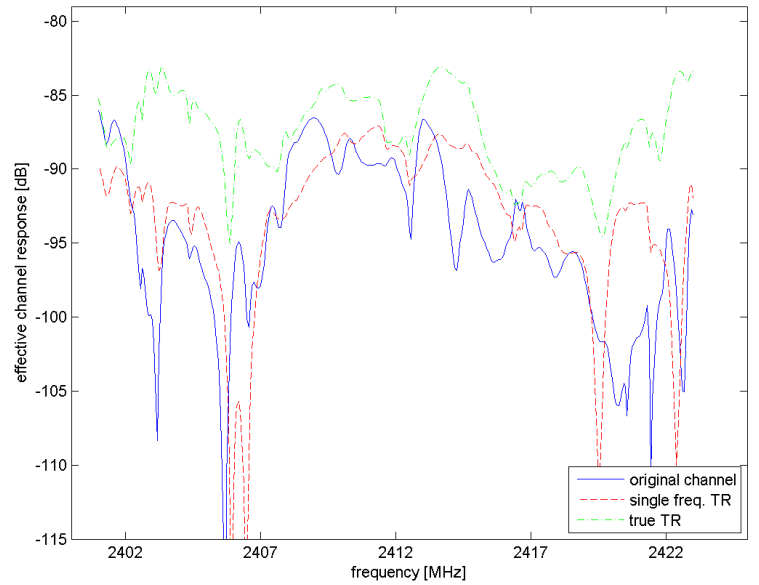

Fig. 3. Frequency Responses for Antenna Array Excited by Equal Amplitude Coefficients, Single Frequency Coefficients and for Ideal Time-Reversal Coefficients.

It is quite surprising to note that the ideal timereversal frequency response significantly differs from the single-frequency time-reversal frequency response. However, there is some similarity in the two responses over the entire range. As seen in the figure the peaks and nulls of the single frequency time-reversal response seem to occur at many of the same frequencies as for the ideal time-reversal frequency response. Despite the fact that the single frequency time-reversal settings are only equivalent to ideal time-reversal over a very narrow range, the time-reversal coefficient has some effect over a wide range. 


\section{MIMO Time-Reversal Focusing}

We have also performed similar measurements for both APs operating on the same channel simultaneously. We have used the measurements of the ventilation duct frequency responses from each of the four AP side antennas to the two user antennas to create a 2 by 2 , time-reversal based, MIMO channel. This was done by applying single frequency time-reversal coefficients that would focus power onto one user location into one set of vector modulators and loading coefficients to focus on the other user location into the second set of modulators. The frequency responses formed in this way were calculated from the originally measured duct channel matrix using the equation

$$
R=k H H^{\dagger}
$$

where $H^{\dagger}$ is the conjugate transpose of the duct channel matrix, $H$, and $k$ is a power correction factor to accommodate the gain restrictions of the vector modulators. The above equation considers the antenna array the transmit side so $H$ is a 4 by 2 matrix and $R$ is the resulting MIMO channel matrix and is 2 by 2 . The calculated $R$ channels are shown in Fig. 4. The figure shows frequency responses for the intended communications paths, $R_{11}$ and $R_{22}$ as well as the cross-talk channels $R_{12}$ and $R_{21}$.
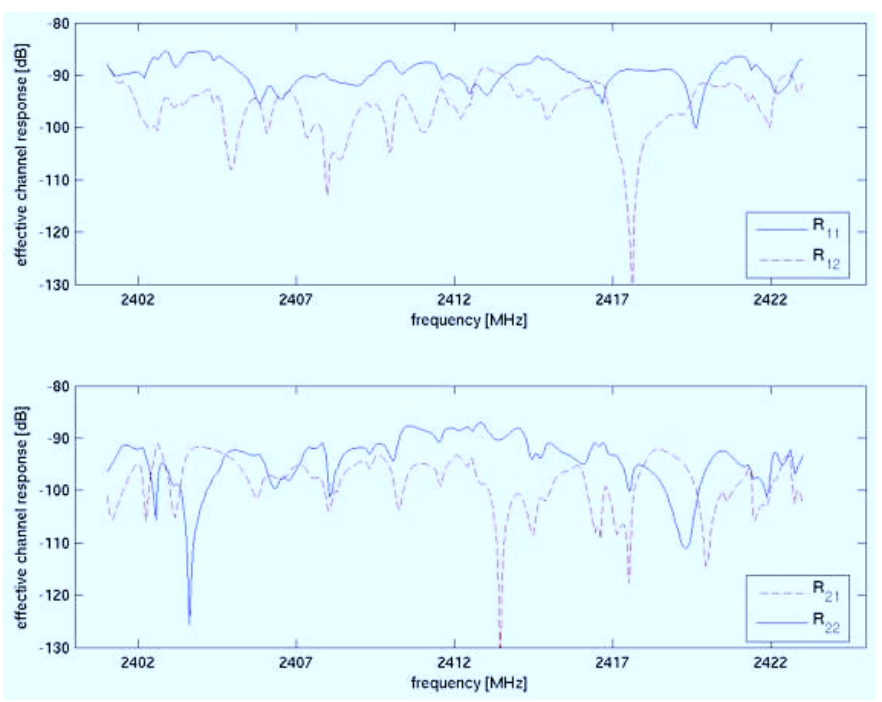

Fig. 4. Signal and interference plots for the 2 by 2 MIMO created communications channels in the ventilation duct network.

The time-reversal coefficients applied to the vector modulators shown in Fig. 1 allow transmissions from $\mathrm{AP}_{1}$ to add coherently to the user location of Laptop 3 , while the transmissions from $\mathrm{AP}_{2}$ should add coherently to the user location of Laptop 4 . To test this, we again measured throughput from Laptop 1 to Laptop 3 . However, we also wished to transmit over both links simultaneously, so we also measured throughput from Laptop $_{2}$ to Laptop 4 . Thus, we measured the throughput for each AP transmitting individually and for both APs transmitting simultaneously. As a baseline, we measured throughput for equal amplitude, zero phase coefficients and for time-reversal coefficients applied to the antenna array elements.

We again used the Iperf [9] throughput measurement software to measure uplink and downlink throughput. Throughput measurements of each link individually were performed using a 60 second measurement duration as before. However, it is difficult to perform throughput measurements exactly simultaneously. Thus, for the simultaneous transmission measurements, the throughput test for $A P_{2}$ was run for a duration of 2 minutes and started first. The throughput test for $A P_{1}$ was started about 10 seconds later and configured to last for 1 minute. Both throughput measurements were of the TCP throughput using a TCP window size of 1 Megabyte. Again we have measured the throughput in the case of equal amplitude and single frequency timereversal coefficient excitations.

The throughput measurement results are presented in Table II. The table presents the measurement results across several dimensions. First, we note that in all cases the time-reversal coefficients have resulted in an increase in throughput over the equal amplitude coefficients. We also notice that when transmissions are performed simultaneously, throughput decreases in all circumstances as would be expected. However, if we consider the total capacity of both APs, some interesting results emerge. In Table II, we have computed the mean throughput of the individual transmissions over the two APs and the sum of the throughput of both APs for the simultaneous transmissions. The direct comparison of these statistics is a way to compare the total throughput available to the AP. In this manner, it is apparent that for the uplink measurements, the interference has reduced the total capacity available from $1.95 \mathrm{Mbps}$ to $1.6 \mathrm{Mbps}$ when equal amplitude coefficients are used. On the other hand, the time-reversal coefficients actually enabled the AP to increase the available throughput from $2.6 \mathrm{Mbps}$ to 3.2 Mbps. This result is not born out in the downlink direction, however. In the downlink direction the total capacity for both the equal amplitude and the timereversal coefficients has been reduced from 1.8 to 0.5 Mbps and from 2.5 to $1.9 \mathrm{Mbps}$ respectively. However, in the equal amplitude case, transmissions were not possible at all for one of the two users whereas the timereversal coefficients have allowed both users to transmit.

It is not clear why the time-reversal coefficients have enabled an increase in capacity in the uplink direction but not the downlink direction. It may be due to the imbalance in the measurement setup in that the APs can not detect each others transmissions but the user NICs can detect and defer to transmissions from both APs and each other. Regardless, the above throughput measurements indicate that time-reversal may be a useful tech- 
TABLE II

THROUGHPUT MEASUREMENTS OF IEEE 802.11G TRANSMISSION PERFORMANCE USING MIMO TIME-REVERSAL ARRAYS. ALL DATA IS PRESENTED IN MBPS.

\begin{tabular}{|c|c|c|c|c|c|c|c|c|}
\hline \multicolumn{9}{|c|}{ Individual Transmissions } \\
\hline & \multicolumn{4}{|c|}{ Uplink } & \multicolumn{4}{|c|}{ Downlink } \\
\hline & \multicolumn{2}{|c|}{ Equal Amplitude } & \multicolumn{2}{|c|}{ Time-Reversal } & \multicolumn{2}{|c|}{ Equal Amplitude } & \multicolumn{2}{|c|}{ Time-Reversal } \\
\hline & AP 1 & AP 2 & AP 1 & AP 2 & AP 1 & AP 2 & $\mathrm{AP} 1$ & $\mathrm{AP} 2$ \\
\hline & 3.5 & 0.4 & 3.7 & 1.4 & 2.9 & 0.6 & 3.4 & 1.5 \\
\hline Mean & \multicolumn{2}{|c|}{1.95} & \multicolumn{2}{|c|}{2.6} & \multicolumn{2}{|c|}{1.8} & \multicolumn{2}{|c|}{2.5} \\
\hline \multicolumn{9}{|c|}{ Simultaneous Transmissions } \\
\hline & \multicolumn{4}{|c|}{ Uplink } & \multicolumn{4}{|c|}{ Downlink } \\
\hline & \multicolumn{2}{|c|}{ Equal Amplitude } & \multicolumn{2}{|c|}{ Time-Reversal } & \multicolumn{2}{|c|}{ Equal Amplitude } & \multicolumn{2}{|c|}{ Time-Reversal } \\
\hline & AP 1 & AP 2 & AP 1 & AP 2 & AP 1 & AP 2 & $\mathrm{AP} 1$ & $\mathrm{AP} 2$ \\
\hline & 1.3 & 0.3 & 2.1 & 1.1 & 0 & 0.5 & 0.6 & 1.3 \\
\hline Total & \multicolumn{2}{|c|}{1.6} & \multicolumn{2}{|c|}{3.2} & \multicolumn{2}{|c|}{0.5} & \multicolumn{2}{|c|}{1.9} \\
\hline
\end{tabular}

nique for increasing the capacity available to an AP in a single location and possibly as a method for reducing the effects of co-channel interference on IEEE 802.11g APs. Additionally, this technique may be able to reduce the network latency for wireless communications channels when multiple user transmissions take place by allowing users to transmit data simultaneously. Validation of this idea, however, is beyond the scope of this work.

\section{CONCLUSION}

The above work has explored the impact of timereversal based, multi-element arrays on the actual transmissions of IEEE $802.11 \mathrm{~g}$ signals. We have demonstrated that in MISO configurations, an increase in throughput is readily apparent, even for practically constrained, single frequency time-reversal array coefficients. We have also demonstrated that this improvement in throughput is apparent when MIMO array coefficients are applied. We demonstrated that while the total capacity available to two APs operating simultaneously can be improved using time-reversal arrays, it is not necessarily always the case. However, the potential use of time-reversal antenna arrays for reducing co-channel, and perhaps even adjacent channel interference, has been identified. Lastly, we have also shown that ventilation duct channels could benefit from the use of time-reversal antenna arrays.

\section{REFERENCES}

[1] G. Lerosey, J. de Rosny, A. Tourin, A. Derode, G. Montaldo, and M. Fink, "Time reversal of electromagnetic waves," Physical Review Letters, vol. 92, p. 193904, 14 May 2004. [Online]. Available: http://link.aps.org/abstract/PRL/v92/e193904

[2] J. Tuovinen, G. Shiroma, W. Forsyth, and W. Shiroma, "Multipath communications using a phase-conjugate array," in IEEE MTT-S International Microwave Symposium Digest, vol. 3, Jun. 2003, pp. 1681- 1684

[3] B. E. Henty and D. D. Stancil, "Multipath-enabled super-resolution for $\mathrm{rf}$ and microwave communication using phase-conjugate arrays," Physical Review Letters, vol. 93, no. 24, p. 243904, Dec. 2004.

[4] P. Kyritsi, G. Papanicolaou, P. Eggers, and A. Opera, "Miso time reversal and delay spread compression for fwa channels at 5ghz," Antennas and Wireless Propagation Letters, vol. 3, pp. 96-99, 2004.

[5] P. Kyritsi, G. Papanicolaou, P. Eggers, and A. Oprea, "Time reversal techniques for wireless communications," in Vehicular Technology Conference, vol. 1, Sep. 2004, pp. 47- 51.

[6] H. T. Nguyen, J. B. Andersen, G. F. P. P. Kyritsi, and P. C. F. Eggers, "Time reversal in wireless communications: A measurement-based investigation," IEEE Transactions on Wireless Communications, vol. 5, no. 8, pp. 2242-2252, Aug. 2006.

[7] H. Andersson, P. Larsson, and P. Wikstrom, "The use of hvac ducts for wcdma indoor solutions," in IEEE 59th Vehicular Technology Conference, vol. 1. IEEE, May 2004, pp. 229- 233.

[8] D. Stancil, O. Tonguz, A. Xhafa, A. Cepni, P. Nikitin, and D. Brodtkorb, "High-speed internet access via hvac ducts: a new approach," in Globecom '01. IEEE Global Telecommunications Conference, vol. 6, San Antonio, TX, USA, 2001, pp. 3604-3607.

[9] "Nlanr/dast : Iperf 1.7.0 - the tcp/udp bandwidth measurement tool," http://dast.nlanr.net/Projects/Iperf/. [Online]. Available: http://dast.nlanr.net/Projects/Iperf/ 